\title{
Reducing sperm concentration is critical to limiting the oxidative stress challenge in liquid bull semen
}

\author{
C. Murphy, ${ }^{*}$ A. G. Fahey, $†$ A. Shafat, $\ddagger$ and S. Fair ${ }^{* 1}$ \\ *Department of Life Sciences, Faculty of Science and Engineering, University of Limerick, Limerick, Ireland \\ †School of Agriculture and Food Science, University College Dublin, Dublin 4, Ireland \\ ‡Department of Physiology, National University of Ireland, Galway, Ireland
}

\begin{abstract}
Because of the short breeding season, the use of liquid bull semen is a viable option in seasonal grass-based dairy systems such as Ireland. Currently in Ireland, liquid bull semen contains approximately 5 million sperm per insemination dose and is used within $2.5 \mathrm{~d}$ of collection. The hypothesis of this study was that reducing the sperm number per insemination dose would enable bull sperm to be stored for longer. Semen was collected at a commercial AI center and diluted to 1 (T1), 2 (T2), 3 (T3), 4 (T4), and 5 (T5) million sperm per 0.25 -mL dose in caprogen diluent. On d 0.25 (6 h postcollection), 1, 2, 3, 4, and 5 postcollection, viability, oxidative stress, and mitochondrial activity were assessed using flow cytometry and the fluorescent probes propidium iodide, $\mathrm{CM}-\mathrm{H}_{2} \mathrm{DCFDA}$, and rhodamine 123 , respectively. On the same days, glucose consumption, total antioxidant capacity, and progressive linear motility were assessed. We observed an effect of day and treatment on sperm cell viability, with the highest percentage live found in $\mathrm{T} 1$ and the lowest in $\mathrm{T} 5$ on all days. Oxidative stress in live sperm increased with duration of storage and was affected by treatment, being highest in T5 and lowest in T1 on all days (d 5: $56.4 \pm$ $2.76 \%$ and $28.8 \pm 1.22 \%$, respectively; mean \pm SEM). Both the total antioxidant capacity and percentage of live sperm positive for rhodamine 123 were unaffected by treatment. The concentration of glucose in caprogen declined with time and was lowest in T5 and highest in $\mathrm{T} 1$ on $\mathrm{d} 5$. In conclusion, higher concentrations of sperm have detrimental effects on sperm cell viability and increase oxidative stress but have no effect on the mitochondrial activity of sperm.
\end{abstract}

Key words: sperm, dairy bull, oxidative stress, reactive oxygen species

Received December 14, 2012.

Accepted March 13, 2013.

${ }^{1}$ Corresponding author: sean.fair@ul.ie

\section{INTRODUCTION}

With the abolition of milk quotas within the European Union now imminent (March 31, 2015), the Irish dairy industry has set a target of a $50 \%$ increase in dairy output by the year 2020 (Department of Agriculture, 2010). The underlying basis for this is that Ireland (like New Zealand) has a seasonal grass-based milk production system, which has a significant cost advantage in the form of low-cost milk production. This production system is highly dependent on excellent reproductive performance with compact calving to coincide with the start of the grass growing season (Dillon et al., 1995). Although milk production per cow has increased since the 1970s (Foote, 1996), reproductive performance has dramatically declined (Walsh et al., 2011). To combat this decline in fertility, Ireland, through the Irish Cattle Breeding Federation (ICBF), has implemented an Economic Breeding Index (Berry et al., 2005). This is a profit index that enables farmers to select the most elite and profitable bulls to breed replacement heifers, which will have an increased ability to produce more milk solids per cow per year and an increased ability to become pregnant. Therefore, the intensive use of bulls with a high Economic Breeding Index is the best way to optimize profitability by reversing the fertility problems in the Irish national dairy herd while facilitating increases in milk production (McCarthy et al., 2007).

In 2009, Ireland began to select dairy bulls for use in AI programs using genomic selection so as to reduce the generation interval with greater reliability and increase the rate of genetic gain (Taş et al., 2007; Berry and Kearney, 2011). Potential AI bulls are now being identified within weeks of birth and are entering into AI programs at approximately 10 mo of age, when demand for their semen far exceeds supply. Although these young bulls ( $<12$ mo of age) have undergone puberty, they yield a low volume of semen, which can normally only be collected once per week (Helbig et al., 2007; R. Monaghan, National Cattle Breeding Centre, Enfield, Co. Meath, Ireland; personal communication) compared with 3 times per week for a mature bull. Liquid semen provides an opportunity to maximize the 
number of insemination doses from these elite bulls; however, the industry must devise a protocol to optimize its use.

Liquid semen accounts for $5 \%$ of the total AI usage within the Irish dairy industry, but this can increase to $25 \%$ during the peak dairy breeding season (Al Naib et al., 2011b). Given that $60 \%$ of all dairy inseminations in Ireland occur in a 6-wk period in April and May, the extensive use of fresh semen during the peak breeding season is a viable option, whereby the number of insemination doses per ejaculate could be increased up to 10-fold compared with frozen semen (Verberckmoes et al., 2005). Currently in Ireland, the norm is to include 20 million sperm in each frozen semen dose (Al Naib et al., 2011a), whereas 5 million sperm are included in each liquid dose, which, combined with its lower processing costs, makes liquid semen an attractive option. However, liquid semen is not used beyond $3 \mathrm{~d}$ of collection because of concerns over declining fertility with the use of aged semen (Vishwanath and Shannon, 2000).

The hypothesis of this study was that storing liquid semen at lower sperm concentrations than usual (5 million sperm per dose) would reduce levels of reactive oxygen species (ROS), preserving the antioxidants in the extender and metabolic activity of the sperm, and thereby extending the sperm lifespan. In addition, lowering the sperm number per dose would maximize the use of both young and mature elite sires during the peak dairy breeding season. The objective of this study was to examine the effect of reducing the sperm number per insemination dose, for up to $6 \mathrm{~d}$ postcollection, on a range of in vitro parameters; namely, progressive linear motility (PLM), viability, mitochondrial activity, oxidative stress, total antioxidant capacity (TAC), and glucose consumption.

\section{MATERIALS AND METHODS}

\section{Experimental Design}

Semen was collected from Holstein, British Friesian, Belgian Blue, and Hereford bulls, at the National Cattle Breeding Centre, and the effect of reducing the number of sperm per insemination dose was examined on a range of in vitro parameters. The experiment included 6 collections, with semen collected from 3 to 4 bulls in each collection (collection $=$ replicate). Different bulls were used for most but not all collections, and 12 bulls in total were used over the 6 collections. The minimum quality criteria for semen used on the study were ejaculates with $>500$ million sperm per $\mathrm{mL}$ and with greater than $75 \%$ progressively motile sperm. The sperm concentration of each ejaculate was assessed using a photometer
(IMV Technologies, L'Aigle, France) and was initially diluted in 20\% egg yolk caprogen diluent (1:1), followed by a further dilution in 5\% egg yolk caprogen to give final concentrations (treatments) of 1 (T1), 2 (T2), 3 (T3), 4 (T4) and 5 (T5) million sperm per insemination dose, packaged into straws, as one straw represents one insemination dose (0.25-mL straws; IMV Technologies). Before dilution, the caprogen medium was purged in fresh food-grade nitrogen gas (BOC Gases Ireland, Dublin, Ireland) to dispel oxygen from the medium and create an anaerobic environment to limit the metabolic activity of sperm during liquid storage. Semen from each bull used in a given replicate was separated in a split sample design and equally represented in each treatment. Samples were transported to the laboratory at the University of Limerick (Limerick, Ireland) and stored at ambient temperature $\left(18-20^{\circ} \mathrm{C}\right)$ for up to 6 d postcollection. Four straws from each bull at each concentration were pooled and analyzed for viability, PLM, oxidative stress, and mitochondrial activity, at $6 \mathrm{~h}$ postcollection (referred to as $\mathrm{d} 0.25$ ) and on $\mathrm{d} 1$, $2,3,4$, and 5 . In addition, on each of the assessment days, the contents of 4 straws from each treatment were centrifuged at $10,000 \times g$ at $4^{\circ} \mathrm{C}$ for $10 \mathrm{~min}$, and the supernatant was removed and frozen at $-20^{\circ} \mathrm{C}$ for later batch analysis of glucose concentration and TAC.

\section{PLM}

Progressive linear motility was assessed using a phase contrast microscope (BH-2; Olympus, Center Valley, PA) at a magnification of $400 \times$. A droplet of semen (10 $\mu \mathrm{L}$ ) was placed on a prewarmed slide, covered with a prewarmed coverslip, and assessed by counting 50 motile sperm for each treatment on each assessment day. A sperm cell was deemed to display PLM if it moved in a linear fashion; PLM was expressed as the percentage of live and motile sperm that displayed forward progressive linear motion.

\section{Assessment of Oxidative Stress and Mitochondrial Activity and Viability}

Diluted semen $(350 \mu \mathrm{L})$ from each treatment was washed twice in PBS $(5 \mathrm{~mL})$ at $800 \times g$ at $32^{\circ} \mathrm{C}$ for 10 min. To detect general oxidative stress, the fluorescent probe 5-(and-6)-chloromethyl-2',7'-dichlorodihydrofluorescein diacetate, acetyl ester $\left(\mathrm{CM}-\mathrm{H}_{2} \mathrm{DCFDA}\right.$; Molecular Probes, Eugene, OR) was added to $367 \mu \mathrm{L}$ of the washed sample $\left(1.4 \times 10^{6} \mathrm{sperm} / \mathrm{mL}\right)$ to give a final concentration of $125 \mu M$ and incubated in the dark at $35^{\circ} \mathrm{C}$ for $30 \mathrm{~min}$. Following the incubation period, propidium iodide (PI; Invitrogen, Grand Island, NY) was added to give a final concentration of $50 \mu \mathrm{M}$ and 
the sample was incubated for a further $15 \mathrm{~min}$. Samples were then washed in PBS, as above, to remove excess stain, leaving a $500-\mu \mathrm{L}$ pellet that was transferred to a 5 -mL round-bottomed tube (BD Biosciences, San Jose, CA) and analyzed on a flow cytometer (BD-LSR 1, BD Biosciences). Mitochondrial activity was analyzed as above with the exception of a 15-min incubation period at $35^{\circ} \mathrm{C}$ with rhodamine 123 (R123; Invitrogen) instead of the oxidative stress probe, to give a final concentration of $2.5 \mu \mathrm{M}$. The flow cytometer was furnished with a 488-nm argon ion laser. Fluorescence of both $\mathrm{CM}-\mathrm{H}_{2} \mathrm{DCFDA}$ and R123 probes was read with the FL1 photodetector (530/26 bandpass filter) and PI was read with the FL2 photodetector $(575 / 26$ bandpass filter). Appropriate single-color controls were prepared to establish the respective fluorescent peaks of the individual stains. These were used in conjunction with the forward scatter and side scatter signals to discriminate sperm from debris. Fluorescent events were recorded using CellQuest Pro (version 3.7, BD Biosciences), and all parameters were read using logarithmic amplification. For each sample, 10,000 gated events were captured at approximately 200 events per second. Viability was defined as a percentage of PInegative events, and oxidative stress and mitochondrial activity were defined as the percentage of viable cells positive for the respective stains. All percentages were calculated as part of the total gated sample.

\section{Assessment of Glucose Consumption}

Glucose consumption in the caprogen medium was evaluated using a commercial glucose kit (Megazyme, Bray, Co. Wicklow, Ireland). Supernatant samples were thawed and diluted 1:2 with distilled water, added to $1 \mathrm{~mL}$ of glucose oxidase-peroxidase reagent, and incubated at $45^{\circ} \mathrm{C}$ for 20 min. Absorbance was measured at $510 \mathrm{~nm}$ on a UV-visible spectrophotometer (Cary 100 Bio; Varian, Palo Alto, CA), and glucose concentrations were determined using a glucose standard curve. Glucose concentrations were recorded in micrograms per milliliter of caprogen medium.

\section{Assessment of TAC}

The TAC of the caprogen samples were determined by the 2,2'-azino-bis-3-ethylbenzthiazoline-6-sulfonic acid $\left(\mathbf{A B T S}^{\bullet+}\right)$ radical cation assay using an adapted ABTS $^{\bullet+}$ method (Miller and Rice-Evans, 1997). A commercial antioxidant assay kit (Sigma-Aldrich, Arklow, Co. Wicklow, Ireland), which included ABTS reagent, equine heart myoglobin, Trolox (6-hydroxy-2,5,7,8-tetramethylchroman-2-carboxylic acid), and $3 \%$ hydrogen peroxide $\left(\mathrm{H}_{2} \mathrm{O}_{2}\right)$ was used to determine TAC. Trolox was used for standard antioxidant solutions, ranging from 0 to $0.42 \mathrm{~m} M$, for assay calibration and preparation of a standard curve. Samples were thawed at ambient temperature and centrifuged at $10,000 \times g$ at $20^{\circ} \mathrm{C}$ for $2 \mathrm{~min}$. In a 96 -well plate, myoglobin was added to standards or thawed samples $(2: 1)$, whereas $\mathrm{H}_{2} \mathrm{O}_{2}$ was added to the ABTS substrate to activate it before its addition to each well. The plate was incubated for 5 min at ambient temperature before absorbance was assessed at $405 \mathrm{~nm}$ by using an ultra microplate reader (ELX808IU; BioTek, Winooski, VT). The absorbance of the measured samples was assessed in duplicate with the average value inserted into the equation of the line for the Trolox calibration curve to determine the TAC of the samples, expressed as millimolar Trolox equivalents per liter.

\section{Statistical Analysis}

Data were examined for normality of distribution, tested for homogeneity of variance, transformed where appropriate, and analyzed using the general linear model repeated-measures procedure with a compound symmetry covariance structure in SPSS software (version 20.0, IBM, Chicago, IL). Motility data were transformed using a logarithm transformation, mitochondrial activity using a power transformation (lambda $=2.0$ ), and TAC using a square root transformation. Fixed effects included in the model were treatment, day of storage, and breed of bull; bull was a random effect. However, breed, bull, and their interactions were not significant. The final model included the main effects of treatment, day, and treatment $\times$ day interactions. Post hoc tests were carried out using the Bonferroni test, and results are reported as the mean \pm the standard error of the mean.

\section{RESULTS}

\section{Effect of Sperm Concentration on Viability}

We observed an effect of both day and treatment on viability $(P<0.001$ : Figure 1$)$, with the percentage of live cells in T1 being $92.4 \pm 0.68 \%$ and $80.8 \pm 2.32 \%$ on $\mathrm{d} 0.25$ and $\mathrm{d} 5$, respectively, whereas the percentage of live cells in T5 was $66.4 \pm 2.79 \%$ and $56.1 \pm 3.23 \%$ on $\mathrm{d} 0.25$ and $\mathrm{d} 5$, respectively. In addition, T2 had a greater proportion of viable cells than T4 and T5 $(P$ $<0.05$ and 0.01 , respectively). Although viability was assessed only at $6 \mathrm{~h}$ postcollection (d 0.25), we observed an unexpected difference between treatments in the percentage of viable sperm. Treatment $\mathrm{T} 1$ yielded the greatest percentage of viable sperm compared with all other treatments $(P<0.01)$, with these initial differ- 


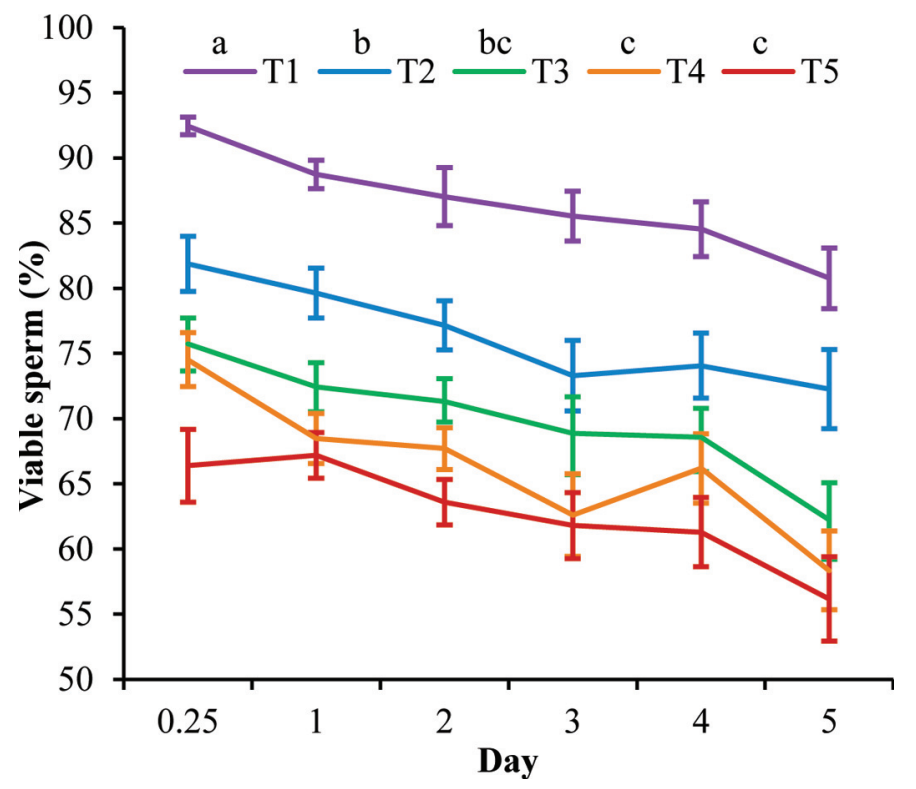

Figure 1. The effect of sperm concentration per 0.25 -mL insemination dose on viability as assessed on d $0.25,1,2,3,4$, and 5 . Vertical bars represent SEM. ${ }^{a-c}$ Letters represent significant differences between treatments. Color version available in the online PDF.

ences remaining throughout the duration of the experiment.

\section{Effect of Sperm Concentration on PLM}

From d 0.25 to 5 , the percentage of motile sperm displaying PLM declined linearly $(P<0.001$; Figure 2$)$. However, treatment did not affect PLM, with T1 having a PLM of $94.9 \pm 1.57 \%$ and $72.3 \pm 2.26 \%$ on d 0.25 and 5 , respectively, compared with $\mathrm{T} 5$, which yielded a PLM of $95.3 \pm 1.60 \%$ and $74.4 \pm 2.13 \%$ on $\mathrm{d} 0.25$ and d 5 , respectively. Hence, treatment or treatment $\times$ day interactions had no overall effect on PLM $(P>0.05)$; however, all treatments in the study retained a PLM score of $>50 \%$ up to $5 \mathrm{~d}$ postcollection.

\section{Effect of Sperm Concentration on Mitochondrial Activity and Oxidative Stress}

We found no treatment $x$ day interaction for mitochondrial activity or oxidative stress. The percentage of live cells positive for mitochondrial activity ranged between $94.8 \pm 0.53 \%$ and $91.9 \pm 0.63 \%$ over the duration of storage and was not affected by treatment or treatment $x$ day interactions. The levels of oxidative stress in each treatment increased with respect to storage day $(P<0.001$ : Figure 3$)$. The percentage of viable sperm positive for oxidative stress were $12.4 \pm 1.39 \%$ and $28.8 \pm 1.22 \%$ for $\mathrm{T} 1$ on d 0.25 and 5 , respectively, and $30.8 \pm 4.35 \%$ and $56.4 \pm 2.76 \%$ for $\mathrm{T} 5$ on $\mathrm{d} 0.25$ and 5, respectively. We also found an effect of treatment $(P<0.001)$, with $\mathrm{T} 1$ having a lower percentage of viable sperm staining positive for oxidative stress than all other treatments $(P<0.01)$. Similarly, T2 had a lower proportion of viable sperm positive for oxidative stress than both T4 and T5 $(P<0.01)$.

\section{Effect of Sperm Concentration on Glucose Consumption and TAC}

We found no treatment $x$ day interaction for glucose consumption or TAC. Glucose concentrations in caprogen medium declined over the duration of storage $(P$ $<0.001)$, yielding values of $1,080.4 \pm 26.17$ and 964.4 $\pm 21.61 \mu \mathrm{g} / \mathrm{mL}$ for $\mathrm{T} 1$ on $\mathrm{d} 0.25$ and 5 , respectively, and $1,002.6 \pm 24.61$ and $831.3 \pm 22.25 \mu \mathrm{g} / \mathrm{mL}$ for $\mathrm{T} 5$ on d 0.25 and 5 , respectively (Figure 4). We observed an effect of treatment $(P<0.01)$, with T1 having the highest concentration of glucose on d 5 and T5 having the lowest $(P<0.01)$. Interestingly, over the duration of storage, we found that, on average, a single sperm consumed approximately 4 times more glucose in $\mathrm{T} 1$ compared with T5 $(0.12 \pm 0.02$ and $0.03 \pm 0.01 \mathrm{ng} / \mathrm{mL}$ of glucose, respectively). Treatment had no effect on TAC, with treatments ranging between 0.16 and 0.24 $\mathrm{m} M$ Trolox equivalents $(P>0.05)$. However, although day had an effect on TAC, we found no clear pattern of biological significance.

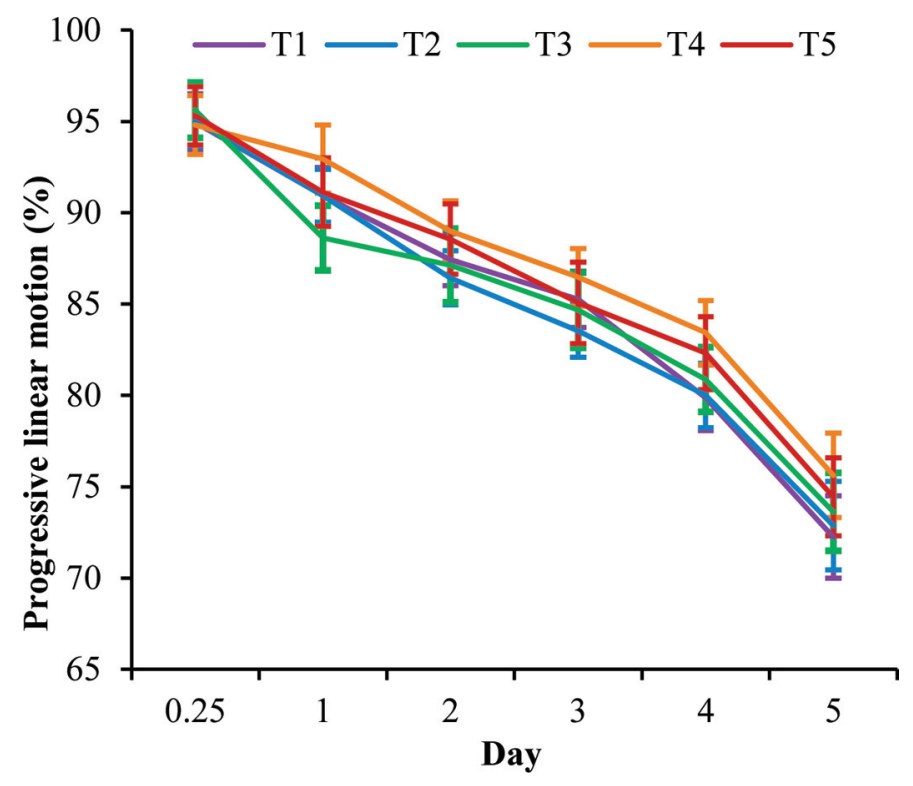

Figure 2. The effect of sperm concentration per $0.25-\mathrm{mL}$ insemination dose on progressive linear motion as assessed on $\mathrm{d} 0.25,1,2,3$, 4, and 5. Vertical bars represent SEM. Color version available in the online PDF. 


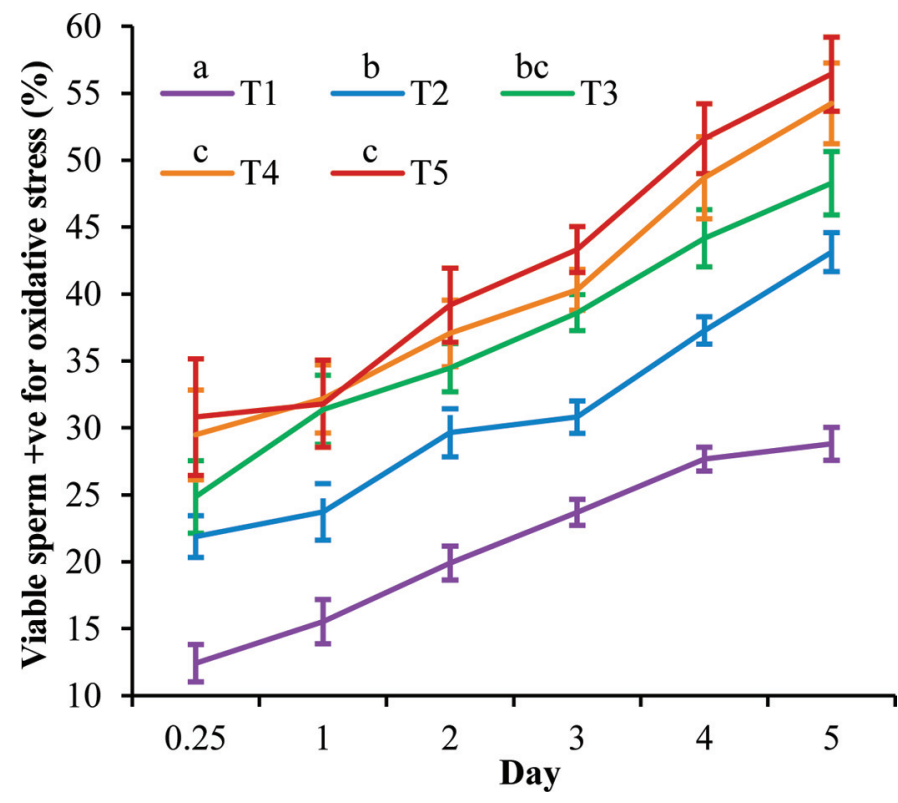

Figure 3. The effect of sperm concentration per $0.25-\mathrm{mL}$ insemination dose on oxidative stress as assessed on d $0.25,1,2,3,4$, and 5 . Vertical bars represent SEM. ${ }^{a-c}$ Letters represent significant differences between treatments. Color version available in the online PDF.

\section{DISCUSSION}

The main findings of this study were that lowering the number of sperm per insemination dose (1) had a significant beneficial effect on sperm cell viability, (2) had a significant beneficial effect on the oxidative status, and (3) did not affect PLM or the mitochondrial activity of sperm of bull sperm stored in vitro for up to 5 d postcollection.

Sperm viability declined in all treatments with respect to time, as was found in previous studies (Krzyzosiak et al., 2001; Verberckmoes et al., 2005). In addition, treatments with lower sperm numbers ( $\mathrm{T} 1$ and $\mathrm{T} 2$ ) retained higher viability from d 0.25 to 5 compared with treatments with higher concentrations ( $\mathrm{T} 4$ and T5). In agreement with this, Prathalingam et al. (2006) found that sperm diluted to 10 million sperm $/ \mathrm{mL}$ and stored at ambient temperature retained higher viability and lower osmotic stress compared with sperm stored at 60 million sperm $/ \mathrm{mL}$. The unexpected differences in viability present on $\mathrm{d} 0.25$ may be linked to a rapid initial peak of the superoxide anion (de Lamirande et al., 1998; Said et al., 2004; Kiernan et al., 2012; not assessed in the current study), a primary ROS that can dismutate either spontaneously or enzymatically to hydrogen peroxide and oxygen (Aitken et al., 1997; de Lamirande et al., 1997; Kowalowka et al., 2008). Therefore, the higher concentrations of sperm may lead to an increased level of dismutation of the superoxide anion, generating higher levels of oxidative stress within the sperm sample (de Lamirande and O'Flaherty, 2008).

Sperm generate energy using both aerobic and anaerobic pathways. One of the by-products from the aerobic pathway are ROS (Oborna et al., 2010) and, although they are involved in cell signaling at low levels, excessive production of ROS can lead to oxidative stress, lipid peroxidation, DNA damage, and associated impairment of sperm functional parameters (Aitken and Baker, 2006; De Iuliis et al., 2009; Linschooten et al., 2011). Using the fluorescent oxidative stress probe, we demonstrated that the levels of ROS produced in each treatment increased during storage, with the higher sperm concentration unexpectedly having an augmented initial oxidative status on d 0.25 , the day of collection, even though sperm were packaged from the same ejaculate. This novel finding indicates that the first $6 \mathrm{~h}$ postcollection may be a critical period during liquid bovine semen storage in terms of reducing the production of ROS and limiting the associated loss of sperm function (Castellini et al., 2003; de Lamirande and Lamothe, 2009). The reduced production of ROS on d 0.25 in the lower sperm concentrations was sustained throughout the storage period, with the oxidative stress levels of T1 on d 5 being comparable to that of $\mathrm{T} 5$ on $\mathrm{d} 0.25$. When sperm are vulnerable to degradation or scavenging by ROS they may become defective and are more likely to generate increasing quantities of ROS, leading to peroxidative damage (Koppers et al., 2008). To combat this, given their limited amount

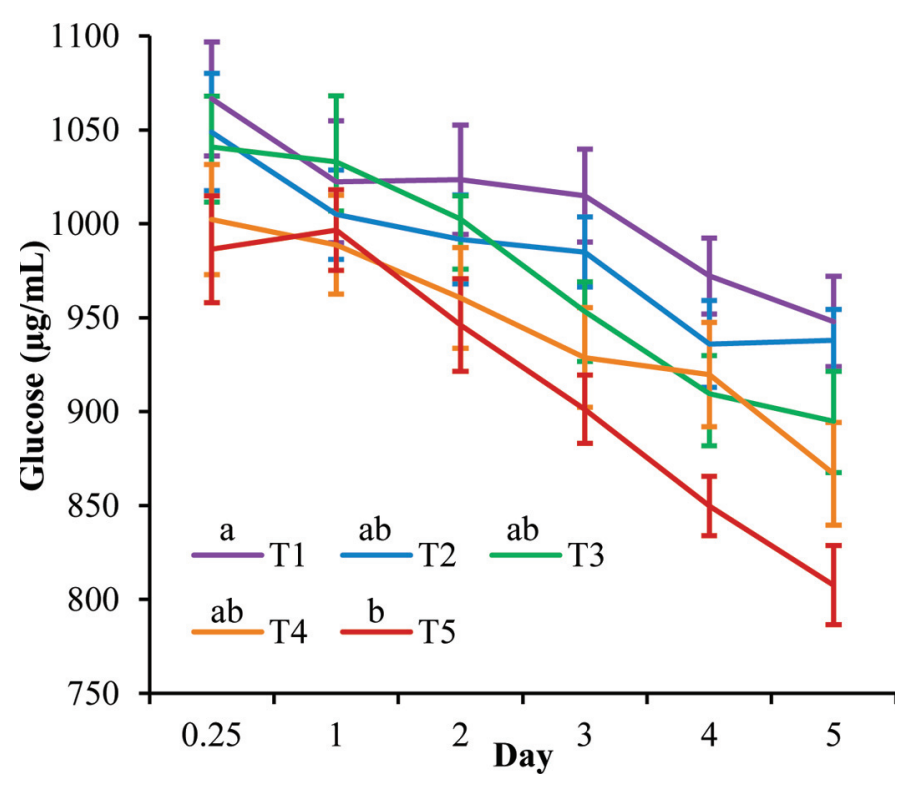

Figure 4. The effect of sperm concentration per $0.25-\mathrm{mL}$ insemination dose on glucose consumption as assessed on d $0.25,1,2,3,4$, and 5. Vertical bars represent SEM. ${ }^{\mathrm{a}-\mathrm{c}}$ Letters represent significant differences between treatments. Color version available in the online PDF. 
of cytoplasm, sperm may rely heavily on antioxidant supplementation in their surrounding media (Kolettis et al., 1999; Castellini et al., 2003; Gliozzi et al., 2009). However, despite the differences in ROS production observed in the current study, the TAC of each of the treatments remained similar throughout storage and showed little variation with respect to treatment or time.

The parameters of PLM, mitochondrial activity, and glucose consumption were assessed to determine an effect of sperm concentration on metabolism. As expected, the PLM score on all treatments declined with respect to time (Verberckmoes et al., 2005); however, all treatments retained a score of at least $50 \%$ up to $\mathrm{d} 5$ of the study. This finding is supported by other studies that noted that bull sperm can retain slowly decreasing PLM scores for up to 4 wk when stored in caprogen despite a sharp decline in nonreturn rates (NRR) from $69.9 \pm 1.2 \%$ after 3 to $5 \mathrm{~d}$ compared with $41.5 \pm 3.7 \%$ after $10 \mathrm{~d}$ (Shannon, 1965). We found no difference in PLM scores between treatments despite differences in ROS levels. In contrast, other studies have reported that human liquid semen samples with higher levels of ROS had impaired sperm motility (Iwasaki and Gagnon, 1992; Armstrong et al., 1999; El-Taieb et al., 2009). In addition, it was recently reported that sperm stored for greater than 3 $\mathrm{d}$ have a reduced ability to penetrate artificial mucus (Al Naib et al., 2011b); however, the assessment of mitochondrial activity in the current study showed that the number of live sperm with active mitochondria remained stable and high throughout the study (ranging from $94.8 \pm 0.53 \%$ to $91.9 \pm 0.63 \%$ ). This indicates that the inability of stored sperm to penetrate artificial mucus is not attributable to sperm mitochondrial activity. Despite storage in anaerobic conditions, sperm were highly metabolically active. This may be attributable to the exposure of sperm to regular atmospheric conditions upon release from the straw and a reactivation of the mitochondria; however, all treatments responded equally and no significant variation was recorded. Over the duration of storage, treatments with lower sperm numbers were found to consume less glucose than treatments with higher concentrations. However, the amount of glucose consumed did not decrease proportionally to the number of cells present in each insemination dose, perhaps because of the viability status of the sperm in each treatment. On an individual sperm basis, it was found that a sperm stored at T1 consumed approximately 4 times more glucose than a sperm stored at T5. This difference may be attributable to the greater number of cells present per straw and storage under $\mathrm{N}$ gas. The decreased oxygen concentration resulting from these factors may cause an accelerated shift in sperm metabolism to the glycolytic pathway and an increased production of lactate, possibly reducing the intracellular $\mathrm{pH}$. (Storey, 2008). It was previously reported that the reduction in fertilizing potential during the prolonged storage of liquid semen was due to substrate exhaustion rather than ROS (Krzyzosiak et al., 2001). However, our results demonstrate that sufficient glucose was present to maintain sperm cells for at least $5 \mathrm{~d}$ postcollection.

Recent studies in ram sperm have indicated that sorted sperm can successfully fertilize in vivo at doses as low as 1 million sperm per insemination dose (de Graaf et al., 2007). In addition, these fertility rates are comparable with those of nonsorted doses at 50 million sperm, supporting the hypothesis that lowering the sperm concentration per dose would not be detrimental to in vivo fertility rates. Similarly in cattle, evidence shows no differences between fresh (when inseminated within $3 \mathrm{~d}$ of collection) or frozen semen in NRR (Shannon and Vishwanath, 1995; Buckley et al., 2003; Berry et al., 2011) or in vitro fertilization rates (Verberckmoes et al., 2005). Field trials carried out in New Zealand (Shannon and Vishwanath, 1995) and the United States (Seidel et al., 1997) have shown NRR of 68 and $62 \%$, respectively, when low-dose liquid semen inseminations $\left(2.5 \times 10^{6}\right)$ were used. Shannon and Vishwanath (1995) also reported differences in NRR between optimum $\left(2.5 \times 10^{6}\right)$ and suboptimum $(0.5 \times$ $10^{6}$ ) insemination doses, with suboptimum reporting an average decrease of 5.5 to $9.1 \%$. This is compared with a reported average NRR of $53 \%$ in Ireland (Buckley et al., 2003), where liquid insemination is performed with approximately 5 million sperm per dose. The above evidence indicates that a field trial must be conducted to establish the effect of a reduction in sperm number on in vivo fertility and to determine the optimum storage concentration for liquid semen.

\section{CONCLUSIONS}

Reducing the sperm number per insemination dose could facilitate not only the increased usage of young genomically selected sires during a short breeding season but also the more prolonged storage of liquid bull semen because of lower levels of oxidative stress. The results of this in vitro study provide a solid rationale for a future field trial to be carried out to investigate whether a reduced liquid sperm concentration would equal or surpass current in vivo fertility rates. Further work is required to elucidate the reasons for the differences in ROS production on the day of collection between sperm stored at differing concentrations and the possible targeted use of antioxidants during this critical period. 


\section{ACKNOWLEDGMENTS}

The authors gratefully acknowledge support from the Irish Research Council (Dublin, Ireland) and Enterprise Ireland (Dublin, Ireland). Semen was donated by the National Cattle Breeding Centre (Enfield, Co. Meath, Ireland).

\section{REFERENCES}

Aitken, R. J., and M. A. Baker. 2006. Oxidative stress, sperm survival and fertility control. Mol. Cell. Endocrinol. 250:66-69.

Aitken, R. J., H. M. Fisher, N. Fulton, E. Gomez, W. Knox, B. Lewis, and S. Irvine. 1997. Reactive oxygen species generation by human spermatozoa is induced by exogenous NADPH and inhibited by the flavoprotein inhibitors diphenylene iodonium and quinacrine. Mol. Reprod. Dev. 47:468-482.

Al Naib, A., J. P. Hanrahan, P. Lonergan, and S. Fair. 2011a. In vitro assessment of sperm from bulls of high and low field fertility. Theriogenology 76:161-167.

Al Naib, A., F. Ward, A. K. Kelly, M. Wade, J. I. Marti, and P. Lonergan. 2011b. Effect of duration of storage at ambient temperature on fertilizing ability and mucus penetration ability of fresh bovine sperm. Theriogenology 76:1070-1075.

Armstrong, J. S., M. Rajasekaran, W. Chamulitrat, P. Gatti, W. J. Hellstrom, and S. C. Sikka. 1999. Characterization of reactive oxygen species induced effects on human spermatozoa movement and energy metabolism. Free Radic. Biol. Med. 26:869-880.

Berry, D. P., R. D. Evans, and S. Mc Parland. 2011. Evaluation of bull fertility in dairy and beef cattle using cow field data. Theriogenology $75: 172-181$.

Berry, D. P., and J. F. Kearney. 2011. Imputation of genotypes from low- to high-density genotyping platforms and implications for genomic selection. Animal 5:1162-1169.

Berry, D. P., L. Shalloo, A. R. Cromie, V. E. Olori, and P. Amer. 2005. Economic breeding index for dairy cattle in Ireland. Accessed Jul. 31，2012. http://www.icbf.com/publications/files/ICBF_Jan05_ EBIreport.pdf.

Buckley, F., J. Mee, K. O'Sullivan, R. Evans, D. Berry, and P. Dillon. 2003. Insemination factors affecting the conception rate in seasonal calving Holstein-Friesian cows. Reprod. Nutr. Dev. 43:543-555.

Castellini, C., P. Lattaioli, A. Dal Bosco, A. Minelli, and C. Mugnai. 2003. Oxidative status and semen characteristics of rabbit buck as affected by dietary vitamin E, C and n-3 fatty acids. Reprod. Nutr. Dev. 43:91-103.

de Graaf, S. P., G. Evans, W. M. Maxwell, J. A. Downing, and J. K. O'Brien. 2007. Successful low dose insemination of flow cytometrically sorted ram spermatozoa in sheep. Reprod. Domest. Anim. 42:648-653.

De Iuliis, G. N., L. K. Thomson, L. A. Mitchell, J. M. Finnie, A. J. Koppers, A. Hedges, B. Nixon, and R. J. Aitken. 2009. DNA damage in human spermatozoa is highly correlated with the efficiency of chromatin remodeling and the formation of 8-hydroxy2 '-deoxyguanosine, a marker of oxidative stress. Biol. Reprod. 81:517-524.

de Lamirande, E., A. Harakat, and C. Gagnon. 1998. Human sperm capacitation induced by biological fluids and progesterone, but not by NADH or NADPH, is associated with the production of superoxide anion. J. Androl. 19:215-225.

de Lamirande, E., H. Jiang, A. Zini, H. Kodama, and C. Gagnon. 1997. Reactive oxygen species and sperm physiology. Rev. Reprod. $2: 48-54$.

de Lamirande, E., and G. Lamothe. 2009. Reactive oxygen-induced reactive oxygen formation during human sperm capacitation. Free Radic. Biol. Med. 46:502-510.

de Lamirande, E., and C. O'Flaherty. 2008. Sperm activation: Role of reactive oxygen species and kinases. Biochim. Biophys. Acta 1784:106-115.
Department of Agriculture. 2010. Food Harvest 2020: A vision for Irish agri-food and fisheries. Accessed Jul. 31, 2012. http://www. agriculture.gov.ie/media/migration/agri-foodindustry/foodharvest 2020/2020FoodHarvestEng240810.pdf.

Dillon, P., S. Crosse, G. Stakelum, and F. Flynn. 1995. The effect of calving date and stocking rate on the performance of springcalving dairy cows. Grass Forage Sci. 50:286-299.

El-Taieb, M. A. A., R. Herwig, E. A. Nada, J. Greilberger, and M. Marberger. 2009. Oxidative stress and epididymal sperm transport, motility and morphological defects. Eur. J. Obstet. Gynecol. Reprod. Biol. 144(Suppl. 1):S199-S203.

Foote, R. H. 1996. Review: Dairy cattle reproductive physiology research and management-Past progress and future prospects. J. Dairy Sci. 79:980-990.

Gliozzi, T. M., L. Zaniboni, A. Maldjian, F. Luzi, L. Maertens, and S. Cerolini. 2009. Quality and lipid composition of spermatozoa in rabbits fed DHA and vitamin $\mathrm{E}$ rich diets. Theriogenology 71:910-919.

Helbig, L., M. R. Woodbury, J. C. Haigh, and A. D. Barth. 2007. The onset of puberty in North American bison (Bison bison) bulls. Anim. Reprod. Sci. 97:12-24.

Iwasaki, A., and C. Gagnon. 1992. Formation of reactive oxygen species in spermatozoa of infertile patients. Fertil. Steril. 57:409-416.

Kiernan, M., A. G. Fahey, and S. Fair. 2012. The effect of the in vitro supplementation of exogenous long chain fatty acids on bovine sperm cell function. Reprod. Fertil. Dev. http://dx.doi. org/10.1071/RD12204.

Kolettis, P. N., R. K. Sharma, F. F. Pasqualotto, D. Nelson, A. J. J. Thomas, and A. Agarwal. 1999. Effect of seminal oxidative stress on fertility after vasectomy reversal. Fertil. Steril. 71:249-255.

Koppers, A. J., G. N. De Iuliis, J. M. Finnie, E. A. McLaughlin, and R. J. Aitken. 2008. Significance of mitochondrial reactive oxygen species in the generation of oxidative stress in spermatozoa. J. Clin. Endocrinol. Metab. 93:3199-3207.

Kowalowka, M., P. Wysocki, L. Fraser, and J. Strzezek. 2008. Extracellular superoxide dismutase of boar seminal plasma. Reprod. Domest. Anim. 43:490-496.

Krzyzosiak, J., P. Molan, L. McGowan, and R. Vishwanath. 2001. Effect of sperm number and oxygenation state of the storage media on in vitro fertility of bovine sperm stored at ambient temperature. Theriogenology 55:1401-1415.

Linschooten, J. O., J. Laubenthal, E. Cemeli, A. Baumgartner, D. Anderson, V. E. Sipinen, G. Brunborg, G. R. Haenen, E. Fthenou, J. J. Briede, F. J. van Schooten, and R. W. Godschalk. 2011. Incomplete protection of genetic integrity of mature spermatozoa against oxidative stress. Reprod. Toxicol. 32:106-111.

McCarthy, S., B. Horan, P. Dillon, P. O'Connor, M. Rath, and L. Shalloo. 2007. Economic comparison of divergent strains of Holstein-Friesian cows in various pasture-based production systems. J. Dairy Sci. 90:1493-1505.

Miller, N. J., and C. A. Rice-Evans. 1997. Factors influencing the antioxidant activity determined by the ABTS.+ radical cation assay. Free Radic. Res. 26:195-199.

Oborna, I., G. Wojewodka, J. B. De Sanctis, H. Fingerova, M. Svobodova, J. Brezinova, M. Hajduch, J. Novotny, L. Radova, and D. Radzioch. 2010. Increased lipid peroxidation and abnormal fatty acid profiles in seminal and blood plasma of normozoospermic males from infertile couples. Hum. Reprod. 25:308-316.

Prathalingam, N. S., W. V. Holt, S. G. Revell, S. Jones, and P. F. Watson. 2006. Dilution of spermatozoa results in improved viability following a $24 \mathrm{~h}$ storage period but decreased acrosome integrity following cryopreservation. Anim. Reprod. Sci. 91:11-22.

Said, T. M., A. Agarwal, R. K. Sharma, E. Mascha, S. C. Sikka, and A. J. Thomas. 2004. Human sperm superoxide anion generation and correlation with semen quality in patients with male infertility. Fertil. Steril. 82:871-877.

Seidel, G. E., Jr., C. H. Allen, L. A. Johnson, M. D. Holland, Z. Brink, G. R. Welch, J. K. Graham, and M. B. Cattell. 1997. Uterine horn insemination of heifers with very low numbers of nonfrozen and sexed spermatozoa. Theriogenology 48:1255-1264. 
Shannon, P. 1965. Contribution of seminal plasma, sperm numbers, and gas phase to dilution effects of bovine spermatozoa. J. Dairy Sci. $48: 1357-1361$.

Shannon, P., and R. Vishwanath. 1995. The effect of optimal and suboptimal concentrations of sperm on the fertility of fresh and frozen bovine semen and a theoretical model to explain the fertility differences. Anim. Reprod. Sci. 39:1-10.

Storey, B. T. 2008. Mammalian sperm metabolism: Oxygen and sugar, friend and foe. Int. J. Dev. Biol. 52:427-437.

Taş, M., S. Bacinoglu, U. Cirit, O. B. Ozdas, and K. Ak. 2007. Relationship between bovine fertility and the number of spermatozoa penetrating the cervical mucus within straws. Anim. Reprod. Sci 101:18-27.

Verberckmoes, S., A. Van Soom, J. Dewulf, and A. de Kruif. 2005. Comparison of three diluents for the storage of fresh bovine semen. Theriogenology 63:912-922.

Vishwanath, R., and P. Shannon. 2000. Storage of bovine sperm in liquid and frozen state. Anim. Reprod. Sci. 62:23-53.

Walsh, S. W., E. J. Williams, and A. C. O. Evans. 2011. A review of the causes of poor fertility in high milk producing dairy cows. Anim. Reprod. Sci. 123:127-138. 